\title{
Chemiluminescent solid lipid nanoparticles (SLN) and interations with intact skin
}

Jennifer Breidenich, Julia Patrone, Lisa Kelly, Jason Benkoski, Huong Le, et al.

Jennifer Breidenich, Julia Patrone, Lisa Kelly, Jason Benkoski, Huong Le, Jennifer Sample, "Chemiluminescent solid lipid nanoparticles (SLN) and interations with intact skin," Proc. SPIE 7397, Biosensing II, 73970L (24 August 2009); doi: 10.1117/12.826397

SPIE Event: SPIE NanoScience + Engineering, 2009, San Diego, California, United States 


\title{
Chemiluminescent Solid Lipid Nanoparticles (SLN) and Interactions with Intact Skin
}

\author{
Jennifer Breidenich, Julia Patrone, Lisa Kelly, Jason Benkoski, Huong Le, Jennifer \\ Sample \\ The Johns Hopkins University Applied Physics Laboratory, 11100 Johns Hopkins Rd., \\ Laurel, $M D$
}

\begin{abstract}
We report the synthesis and characterization of a novel nanoparticle formulation designed for skin penetration for the purpose of skin imaging. Solid lipid nanoparticles (SLNs), a drug delivery vehicle, were used as the matrix for targeted delivery of peroxide-sensitive chemiluminescent compounds to the epidermis. Luminol and oxalate were chosen as the chemiluminescent test systems, and a formulation was determined based upon non-toxic components, lotion-like properties, and longevity / visibility of a chemiluminescent signal. The luminescence lifetime was extended in the lipid formulation in comparison to the chemiluminescent system in solution. When applied to porcine skin, our formulation remained detectable relative to negative and positive controls. Initial MTT toxicity testing using HepG2 cells have indicated that this formulation is relatively non-toxic. This formulation could be used to image native peroxides present in tissue that may be indicative of skin disease.
\end{abstract}

\section{INTRODUCTION}

The utilization of nanomaterials for biosensing applications has great potential for early disease detection. Overproduction of hydrogen peroxide occurs in the development of many diseases, including the damage caused to skin by exposure to ultraviolet radiation. Thus, the sensitive detection of low levels of hydrogen peroxide in skin could serve as an early warning indicator for skin cancer. In addition, hydrogen peroxide has been found to be involved in wound healing, ${ }^{1}$ its production is increase by mitochondria during cellular stress response, ${ }^{2}$ and it is of general interest due to its potential involvement in aging processes. Thus we sought to develop a nanomaterials-based biosensor platform capable of detecting hydrogen peroxide present in skin.

Various molecular chemiluminescent systems such as luminol at high $\mathrm{pH}$ and peroxalates combined with a dye are chemically responsive to hydrogen peroxide and can be exploited to indicate its presence, perhaps quantitatively. Luminol is in fact used extensively in assays and as an imaging agent for reactive oxygen species. ${ }^{3}$ However, luminol is not ideal for in vivo detection of peroxides as its optimal $\mathrm{pH}$ is about 10 for luminescence activation. In fact, development of contrast agents for in vivo peroxide detection is an active field of research.

Peroxalates are chemical molecules that, when activated by hydrogen peroxide, can chemically excite the fluorescence of any dye. Thus, this chemistry adds the flexibility of working with various assay emission wavelengths. Peroxalate nanoparticles have been

Biosensing II, edited by Manijeh Razeghi, Hooman Mohseni, Proc. of SPIE Vol. 7397,

73970L · @ 2009 SPIE · CCC code: 0277-786X/09/\$18 - doi: 10.1117/12.826397 
used to image hydrogen peroxide in vivo with high specificity and sensitivity, ${ }^{4}$ and have been prepared with nanomolar sensititivity for intravenous injections. ${ }^{5}$ Thus this system was deemed to be suitable to investigate hydrogen peroxide in skin via solid lipid nanoparticles.

In this study solid lipid nanoparticles used as drug delivery vehicles, serve as the matrix for targeted delivery of chemiluminescent compounds to the epidermis with the goal of detecting native peroxides. As the chemiluminescent agent carrier, solid lipid nanoparticles provide added benefits for using peroxalate chemiluminescence as a diagnostic tool. Their properties are similar to a lotion, so they can easily be applied. They are lipophilic and can therefore interact well with the skin. In addition to stabilizing the loaded material, the SLNs can be used to control the timed release kinetics.
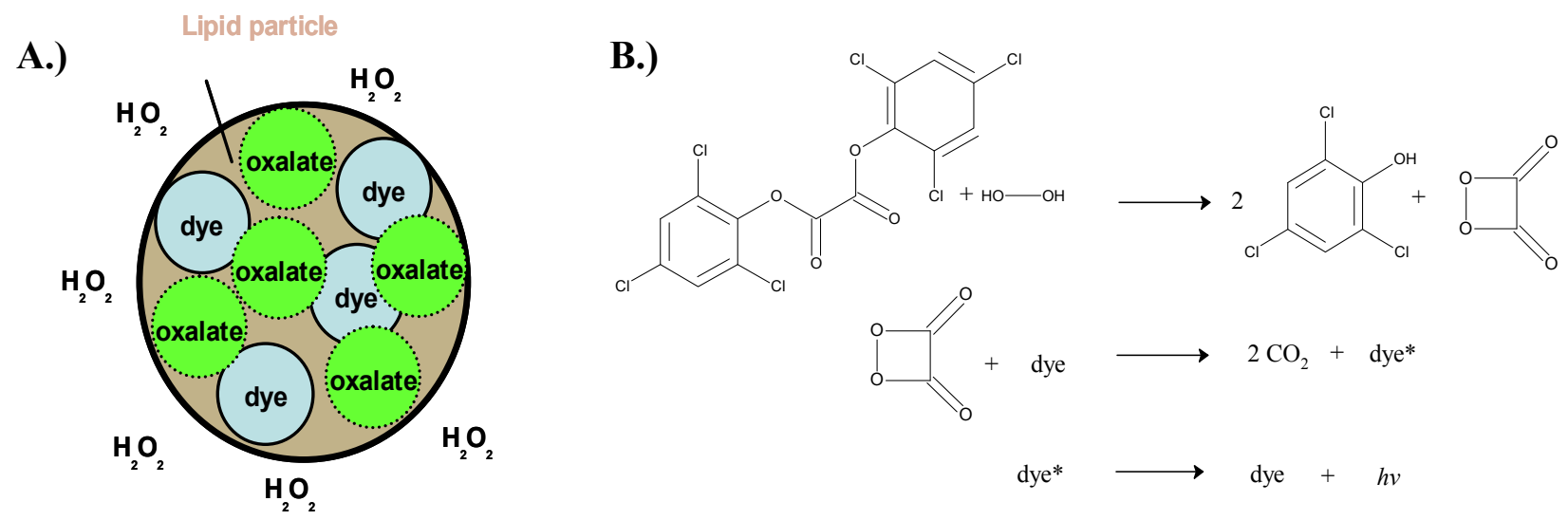

Figure 1. A.) Solid lipid nanoparticle schematic B.) oxalate reaction mechanism ${ }^{6}$

\section{EXPERIMENTAL}

A luminol-loaded solid lipid particle was prepared by the melting method ${ }^{7}$. Stearic acid $(1.0 \mathrm{~g})$ and luminol $(0.1 \mathrm{~g})$ were co-melted at $85^{\circ} \mathrm{C}$. In a separate flask, $0.1 \mathrm{M}$ sodium phosphate $(\mathrm{pH}=11)$, Tween-80 $(1 \mathrm{~mL})$, and potassium hydroxide $(75 \mathrm{mg})$ were stirred, heated to $85^{\circ} \mathrm{C}$, and added dropwise to the lipid mixture. A $10 \%$ (wt. \%) solution of copper ferrocyanide was prepared and added $(50 \mu \mathrm{L})$ to $30 \% \mathrm{H}_{2} \mathrm{O}_{2}(100 \mu \mathrm{L})$. The hydrogen peroxide / copper ferrocyanide solution was added to the SLN to induce chemiluminescence.

An oxalate-loaded solid lipid nanoparticle was synthesized based on the melting method. Cocoa butter (1.6g) was placed in round bottom flask and melted at $70^{\circ} \mathrm{C}$; bis $(2,4,6-$ trichlorophenyl) oxalate $(0.2 \mathrm{~g})$ and the dye, 9, 10-diphenyl anthracene $(0.2 \mathrm{~g})$, were added to the melted cocoa butter and mixed for 10 minutes. The surfactant, $10 \%$ sodium dodecyl sulfate $(2.3 \mathrm{~mL})$, was heated to $70^{\circ} \mathrm{C}$ and added dropwise to the lipid mixture. The water and oil mixture was stirred at $700 \mathrm{rpm}$ overnight and slowly cooled to room temperature. The resulting lotion was stored at $5^{\circ} \mathrm{C}$. 
Hydrogen peroxide detection of the oxalate and dye-loaded SLNs were characterized using a Safire2 microplate reader (Tecan US, Raleigh, NC). Luminescence intensity between the wavelengths of $450 \mathrm{~nm}$ and $600 \mathrm{~nm}$ was recorded for various dyes. Hydrogen peroxide detection was determined by spiking the lotion with $\mathrm{H}_{2} \mathrm{O}_{2}$ and measuring chemiluminescence on the plate reader over time.

In addition to analysis by the plate reader, the chemiluminescence lifetime of this formulation was characterized on pig skin. For the chemiluminescence lifetime experiments, the cooled $4.35 \mathrm{~g}$ of SLN emulsion was spiked with $30 \% \mathrm{wt}$. hydrogen peroxide $(0.67 \mathrm{~mL})$ to give a concentration of $1.15 \mathrm{M} \mathrm{H}_{2} \mathrm{O}_{2}$ in the homogenously mixed lotion. Fresh pig abdominal skin was obtained from a slaughterhouse immediately after slaughter. The skin was cleaned briefly with $70 \%$ ethanol and rinsed in distilled water. Sections of skin were stored at $-80^{\circ} \mathrm{C}$ for no more than 3 months prior to experimentation. A $1.3 \mathrm{~cm} \times 1.3 \mathrm{~cm} \times 0.3 \mathrm{~cm}$ sample of pig skin was loaded with $0.050 \mathrm{~g}$ of $\mathrm{H}_{2} \mathrm{O}_{2}$ spiked lotion and pictures were taken by a Q-Imaging Retiga-4000RV camera in a dark room at an exposure time of 10 seconds.

The particle size was analyzed using dynamic light scattering using a Malvern Zetasizer and and confirmed by optical microscopy using an Olympus BX60M microscope and SEM. DSC was completed using 2960 SDT. SPME GC/MS analysis was performed on a Varian $3800 \mathrm{GC} / \mathrm{MS}$.

Toxicity testing. (Cell culture) Human hepatoblastoma HepG2 cells were cultured in RPMI-1640, supplemented with $1 \mathrm{mM}$ sodium pyruvate, $5 \mathrm{mM}$ HEPES, $2 \mathrm{mM}$ LGlutamine, $10 \%$ fetal bovine serum, $100 \mathrm{U} / \mathrm{ml}$ penicillin, and $100 \mu \mathrm{g} / \mathrm{ml}$ streptomycin. Cells were maintained in a humidified atmosphere at $37^{\circ} \mathrm{C}, 5 \% \mathrm{CO}_{2}$ and passaged at 80

$\%$ confluence. (MTT assay) HepG2 cells were plated at a density of $4 \mathrm{e} 4$ cells/well and allowed to equilibrate at $37^{\circ} \mathrm{C}$ overnight. Lotion stock was diluted in cell culture media as indicated, and $5 \mu \mathrm{l} /$ well was added to each replicate well. After 18 hours of exposure to diluted cocoa butter lotion (containing cocoa butter, SDS, anthracene, and oxalate), cellular health was determined using the MTT assay, according to the manufacturer's protocol. Briefly, MTT reagent was added to the wells of the microplate and after two hours of incubation at $37^{\circ} \mathrm{C}$, intracellular formazin crystals were solubilized with the provided detergent solution. Absorbance values were obtained using the Safire2 microplate reader (Tecan US, Raleigh, NC) with a measurement wavelength of $570 \mathrm{~nm}$ and a reference wavelength of $700 \mathrm{~nm}$, read from the bottom.

\section{RESULTS AND DISCUSSION}

Formula Optimization. Multiple formulations were investigated. Typical formulations reported here consisted of a lipid (matrix), surfactant, which promotes nanoparticle formation, and the active incredient, in this case, the chemiluminescent compounds. Particle structure and behavior is a function of the ingredients and the production conditions. Our initial SLN formulation of stearic acid, Tween, and luminol illustrated that a chemiluminescent agent entrapped in a solid-lipid nanoparticle would provide 
extended luminescence lifetime over luminol in solution consistent with the production of SLN structured materials.

Lipids whose melting temperatures are close to body temperature were chosen, specifically stearic acid and cocoa butter. SLN's studied for drug delivery have shown that the melting point of the lipid and the drug as well as the solubility of the drug in the lipid contribute most to the position of the drug within the SLN. ${ }^{8}$ The lipid component of the SLN was changed from stearic acid to cocoa butter to increase the chemiluminescent signal intensity of the SLN when applied to skin. Solid lipid nanoparticles used for drug delivery rely on diffusion of the drug through the lipid portion of the particle to achieve a timed release. The initial SLN, with stearic acid as the lipid component, gave a lower intensity, extended lifetime chemilumenscent signal at a temperature of $37^{\circ} \mathrm{C}$ during plate reader experiments (data not shown). Benchtop experiments demonstrated that for the stearic acid SLN to be visible by eye, the temperature of the SLN needed to be elevated to $80^{\circ} \mathrm{C}$. To increase the reaction speed and chemiluminescent intensity, the lipid was changed to cocoa butter. At a melting point of $33.5^{\circ} \mathrm{C}$, the chemiluminescent reaction kinetics of the oxalate-loaded SLN on skin rapidly increased upon melting of the SLN. As the SLN becomes liquid, the diffusion rate of the chemiluminescent reagents makes a large, discontinuous jump relative to the diffusivity for solid-state diffusion.

In drug-releasing SLN's, production factors such as surfactant and mixing temperature have an effect on the drug release profile. ${ }^{9}$ The emulsifier plays the role of partitioning the particles within the formulation. As emulsifier is added to the lipid, the coverage of the lipid particle surfaces with surfactant competes with the clustering of lipids to one another. Low molecular weight emulsifiers such as SDS have been shown to rapidly cover lipid surfaces and result in smaller SLNs. ${ }^{10}$ SDS $(150 \mathrm{mM})$ was substituted for Tween because Tween quenched the fluorescence of the dye. Additionally, nonionic surfactants have also been previously shown to quench chemiluminescence ${ }^{11}$; as an anionic surfactant SDS provided the added benefit of not diminishing the chemiluminescent signal.

A formulation based on luminol as the chemiluminescent agent required a high $\mathrm{pH}$ and a toxic catalyst to initiate the chemiluminescent reaction. These two drawbacks necessitated a change to a chemiluminescent system that required milder reaction conditions. Oxalates are known chemiluminescent agents and have recently been incorporated into nanoparticles for in vivo hydrogen peroxide imaging. ${ }^{4}$ The chemiluminescent system formulation components, such as the oxalate, the peroxide, and the dye were optimized to identify the formulation that gave the longest chemiluminescent lifetime within the SLN. Diphenyl oxalate, dinitrophenyl oxalate, and bis(2,4,6 trichlorophenyl) oxalate are known to initiate chemiluminescence in the presence of hydrogen peroxide and a dye. These oxalates were tested, and bis $(2,4,6$ trichlorophenyl) oxalate gave the longest emission lifetime. Lauryl peroxide and benzoyl peroxide were tested as chemiluminescent initiators in SLN formulation. However, only formulations containing hydrogen peroxide exhibitted chemiluminescence. A benefit of the oxalate chemiluminescent system is the ability to 
excite multiple dyes and image a variety of wavelengths. 9, 10-diphenyl anthracene gave the strongest chemiluminescent signal, but rhodamine and 9,10-bis(phenylethynyl) anthracene were also tested and shown to chemiluminesce in the SLN matrix.
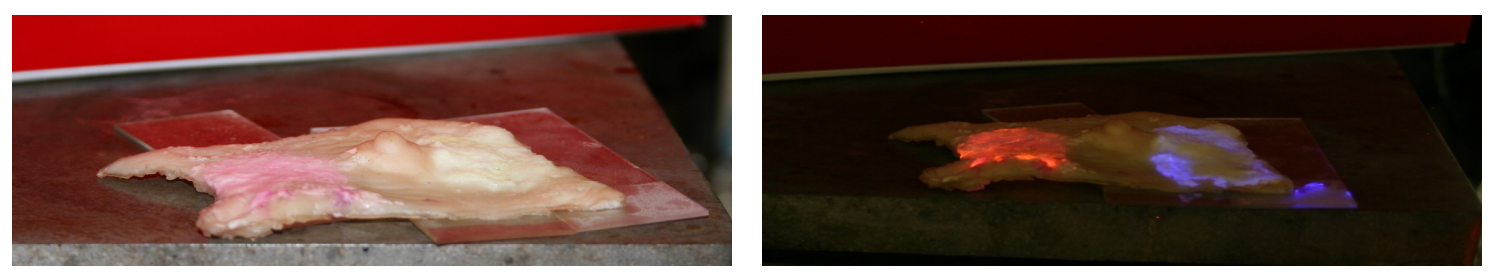

Figure 2. Chemiluminescent SLN lotion made up of Rhodamine (pink) and diphenyl anthracene (blue) on top of pig skin. A.) Photograph of illuminated pig skin B.) Photograph of pig skin in dark room with an exposure time of 4 seconds.

Particle Characterization. Both dynamic light scattering and optical microscopy were used to asses the size and shape of the SLN. Although not sensitive to nanoscale particles, optical microscopy was used to examine SLN shape and homogeneity of the emulsion. Samples were prepared by diluting frozen SLN in cold water and dropping the mixture onto a glass slide. As shown in figure 3, microscopy results illustrated a wide range of sizes and shapes, most likely a result of using the melting method as the SLN preparation method.

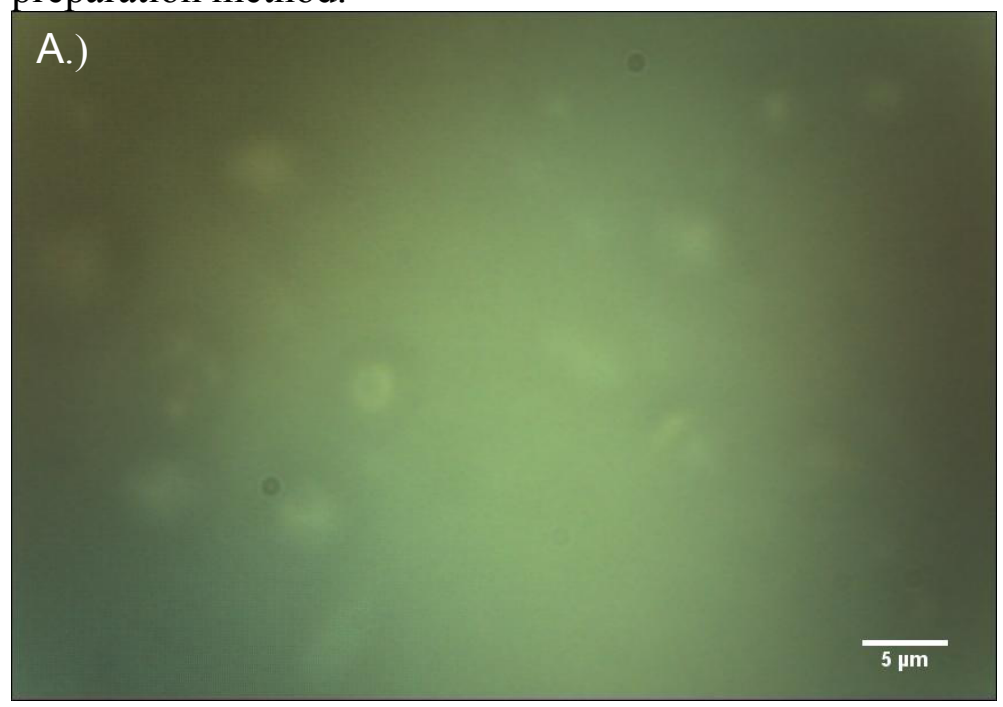




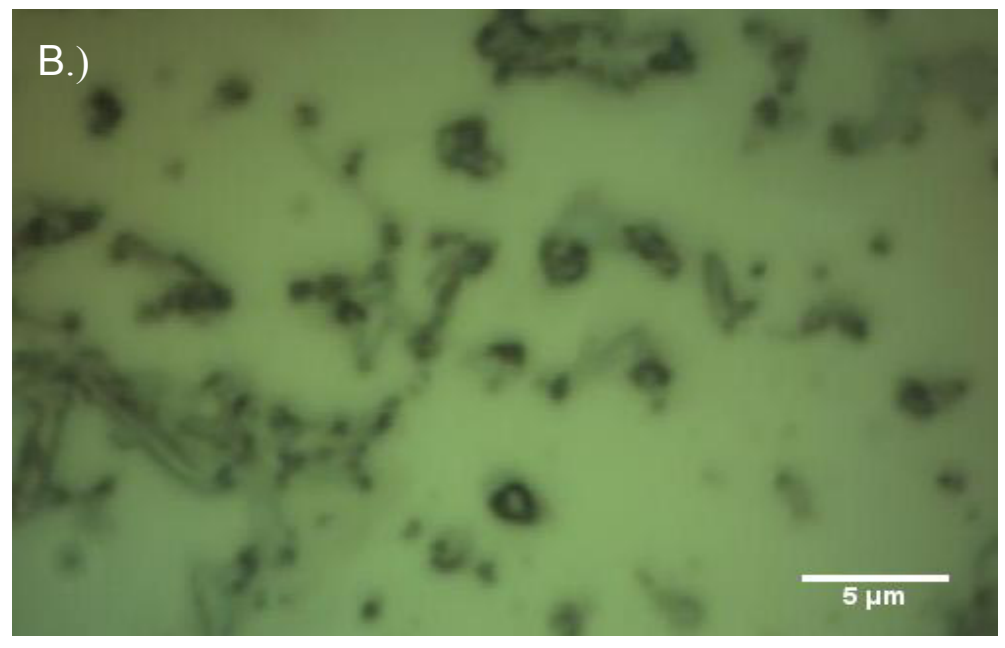

Figure 3. Optical microscope images of the oxalate loaded SLN. A.) Image captured while SLN was dispersed in solution. B.) Image captured following solution evaporation.

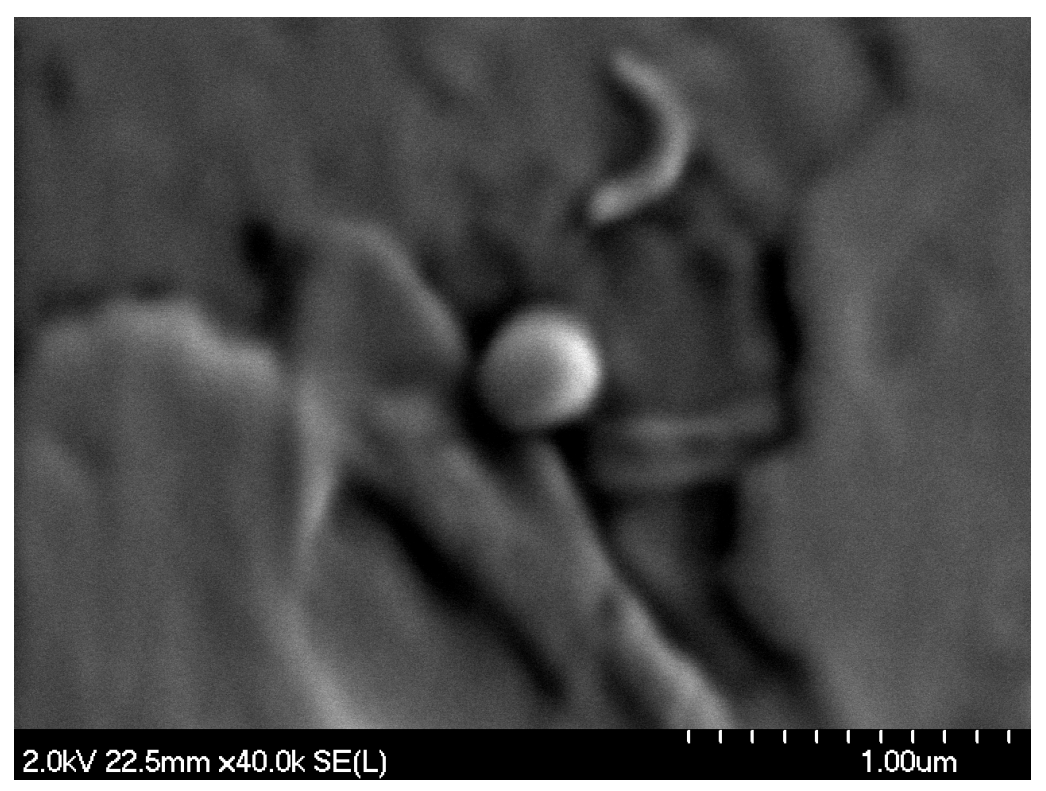

Figure 4. SEM image of SLN evaporated onto a glass slide.

Dynamic light scattering confirmed that the emulsion was a heterogenous mixture of lipid particles. Samples were prepared for the zetasizer analysis by diluting the SLN in water. After filtering through a $1 \mu \mathrm{m}$ filter, dynamic light scattering showed a bimodal size distibution, with the lipid nanoparticles. The average particle diameters were $44.5 \mathrm{~nm}$ and $200 \mathrm{~nm}$ in diameter. Presence of lipid in the solution was confirmed by SPME GC/MS. SEM analysis (figure 4) confirmed a particle diameter of $200 \mathrm{~nm}$. Zeta potential was very stable and remained consistent across all cocoa butter, dye, and oxalate SLNs prepared by 
the melting method at $-77.7 \mathrm{mV}$. This high zeta potential is indicative of decreased particle aggregation due to electric repulsion. ${ }^{9}$

Melting behavior was investigated by differential scanning calorimetry (DSC). The starting material, pure cocoa butter, gave a Gaussian shaped peak at $33.44^{\circ} \mathrm{C}$. Formation of an oxalate-loaded solid lipid nanoparticle led to a melting point depression. The SLN peak was observed at $32.19^{\circ} \mathrm{C}$ and exhibited a shoulder at $31.1^{\circ} \mathrm{C}$.

Hydrogen Peroxide Detection. Effectiveness of the chemiluminescent agent-loaded solid lipid nanoparticle lotion was evaluated by recording the chemiluminescence as a function of time after addition of hydrogen peroxide. Plate reader experiments demonstrated that an oxalate-loaded SLN in the presence of $\mathrm{H}_{2} \mathrm{O}_{2}(29.3 \mu \mathrm{M})$ gave a threefold increase in signal relative to the baseline. Significantly, very little loss of signal was observed during the one hour time period over which data were collected.

\section{Detection of $\mathrm{H}_{2} \mathrm{O}_{2}$}

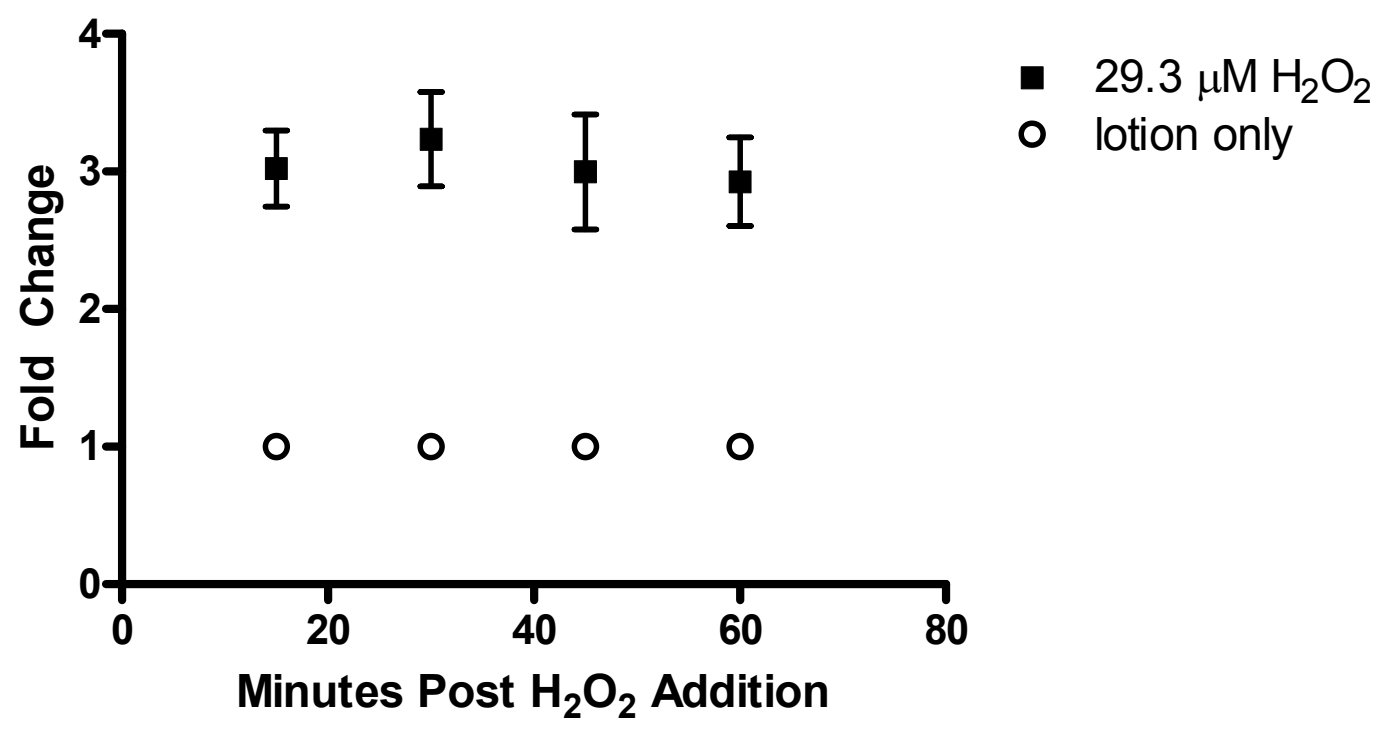

Figure 5. Detection of hydrogen peroxide with cocoa butter SLN containg diphenyloxalate and the anthracene dye. Data shown represents the average of two independent experiments, each performed in triplicate, using separate batches of lotion. Error bars represent the standard deviation of the mean.

Recently synthesized peroxalate micelles have demonstrated a nanomolar sensitivity to hydrogen peroxide when injected intravenously. ${ }^{8}$ Although only micromolar detection of hydrogen peroxide was observed using oxalate-loaded solid lipid nanoparticles, the SLN formulation is still being optimized. However, it is significant to note the longevity of the chemiluminescence that the SLN matrix imparts. We predict that decreasing the particle size will increase the kinetics of chemiluminescence. An increase in intensity is also predicted by increasing the concentration of the oxalate and dye loaded into the particle. Both will improve the detection limit of exogenous hydrogen peroxide. The 
formulation will be optimized to give a chemiluminescent signal lifetime extended long enough for the SLN to be used as a diagnostic tool in a clinical setting and a chemiluminescent signal strength detectable above baseline exogenous hydrogen peroxide concentrations.

In order to investigate SLN behavior on skin, the oxalate-loaded SLN was prepared in the presence of $\mathrm{H}_{2} \mathrm{O}_{2}(1.15 \mathrm{M})$ and transferred onto a pig skin substrate. The samples, along with a blank pig skin control and an oxalate-loaded SLN without $\mathrm{H}_{2} \mathrm{O}_{2}$, on a pig skin substrate were photographed over a one hour period in a dark room at an exposure time of 10 seconds.
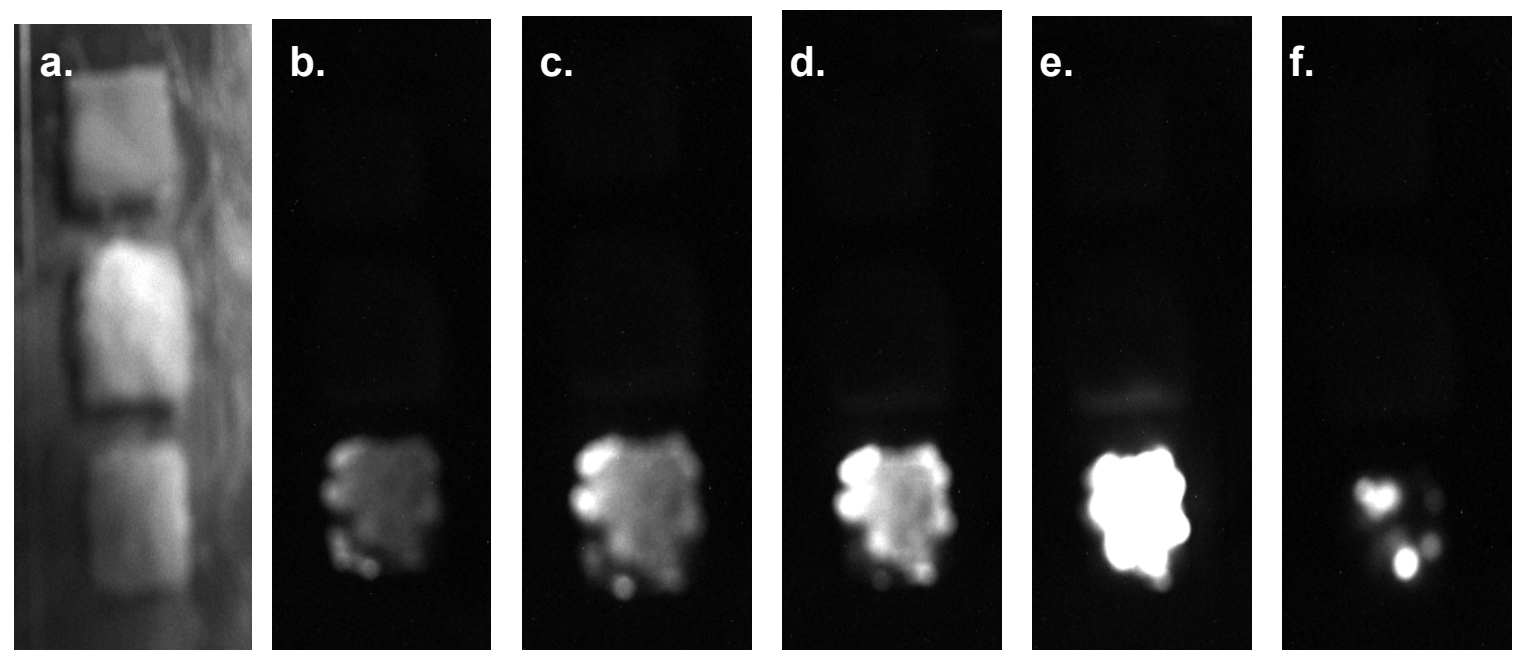

Figure 6. Images of the emulsion applied to pig skin. In frame a, the top square is blank skin, the middle square is skin with cocoa butter SLN, and the bottom square is skin with dye-SLN particles at time $=1$ minute. In b) time $=2 \mathrm{~min}$; c) time $=5 \mathrm{~min} ; \mathrm{d}$ ) time $=$ $10 \mathrm{~min}$; e) time $=30 \mathrm{~min}$; f) time $=1$ hour.

As shown in Figure 5, the camera showed results similar to those observed in the plate reader experiments, with a maximum intensity observed ca. 30 minutes after addition of the hydrogen peroxide. Consistent with data shown in Figure 4, the oxalate-loaded SLN in the presence of hydrogen peroxide gave measurable chemiluminescence over a one hour time period. The pig skin substrate did not appear to dramatically affect the chemiluminescent time release profile.

Toxicity Testing. Initial MTT toxicity testing using HepG2 cells was completed. The formulation was serially diluted and added to cells in a 96-well plate at a 1:20 (volume lotion:volume cells) ratio. The toxicity testing indicated that the formulation is relatively nontoxic, showing no effect on cell viability at levels expected to impact liver tissue. Further MTT toxicity testing will be completed to evaluate the SLN toxicity using primary normal human epidermal keratinocytes (NHEK). 


\section{MTT \\ cocoa butter lotion}

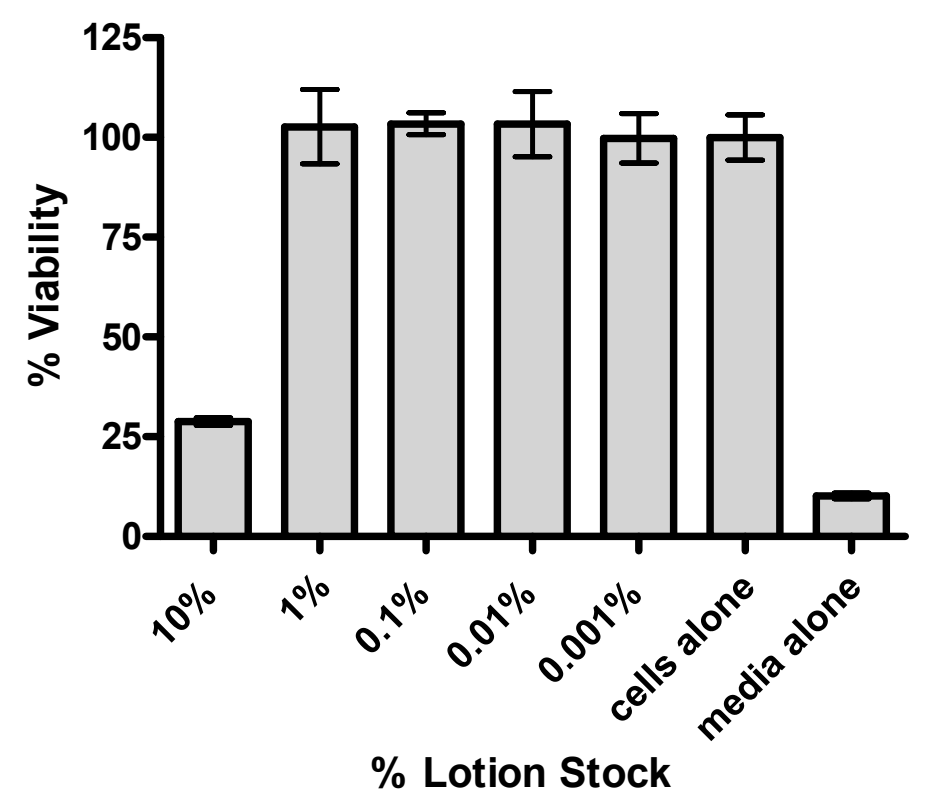

Figure 7. MTT post challenge of HepG2 cells with cocoa butter lotion formulation, including diphenyl oxalate and anthracene dye. Data shown represent one experiment, performed in triplicate and error bars represent the standard deviation of the mean.

Skin Penetrance. A thorough understanding of skin penetrance, localization, and chemiluminescent assay compound metabolism and lifetime will be required to realize the full potential of this formulation as a diagnostic tool. In addition, toxicity testing will be synergistic with skin penetrance studies. Methods to quantify SLN topical absorption by the skin are well documented. Depending on the particle size and primary components, many different pathways through the horny layer of skin are available to the particle. Increased lipophilicity of a particle promotes follicular deposition. SLN's specifically have been demonstrated a fourfold increase in skin absorption over NLC (nanostructured lipid carriers). ${ }^{12}$

Use of a Franz Diffusion Cell to track the SLN through a layer of skin separating the formulation from an acceptor fluid is the most widely accepted evaluation technique. ${ }^{12}$ Once the exposure period is complete; the skin substrate is analyzed by confocal microscopy. These experiments would be suitable next steps for this research.

\section{CONCLUSION}

Chemluminescent agent-loaded SLNs were formulated for chemiluminescent assays and chemiluminescent signal was detectable on a pig skin substrate. The lipid formulation 
increased the longevity of the chemiluminescence signal and enhanced the interaction with the skin, remaining detectable (relative to controls) after application to intact skin.

Acknowledgement This work was supported by internal research and development (IRAD) funding by the Science and Technology Business Area of the Johns Hopkins University Applied Physics Laboratory. We would like to acknowledge Dr. Victor McCrary, the Business Area Executive for Science and Technology for his support of this project. We would also like to thank Mike Rooney, Jill La Favors, and Neil Murphy for their analytical support.

\section{REFERENCES}

${ }^{1}$ Niethammer, P., Grabher, C., Look, A.T., Mitchison, T. J., Nature, 2009, 459, 996.

2 Boveris, A., Chance, B., Biochemical Journal, 1973, 134, 707.

3 Fanger, N. A., Voigtlaender, D., Liu, C., et. al. The Journal of Immunology , 1997, 158,3090 .

${ }^{4}$ Lee, D., Khaja, S., Velasquez-Castano, J. C., et. al. Nature, 2007, 6, 765.

${ }^{5}$ Lee, D., Erigala, V. R., Dasari, M., et. al. International Journal of Nanomedicine, 2008 3(4), 471.

6 Rauhut, M. M., Accounts of Chemical Research, 1969, 2, 80.

${ }^{7}$ Zhang, D., Tan, T., Gao, L., Nanotechnology, 2006, 17, 5821.

${ }^{8}$ Nastruzzi, C. Lipospheres in Drug Targets and Delivery: Approaches, Methods, and Applications, 2005.

9 Müller, R.H., Mäder, K., Gohla, S., European Journal of Pharmaceutics and Biopharmacentics, 2000, 50, 161.

${ }^{10}$ Mehnert, W., Mäder, K., Advanced Drug Delivery Reviews, 2001, 47, 165.

${ }_{11}$ Safavi, A., Karimi, M. A., Analytica Chimica Acta, 2002, 468, 53

${ }^{12}$ Schäfer-Korting, M., Mehnert, W., Korting, H., Advanced Drug Delivery Reviews, 2007, 59, 427. 